\title{
75 Jahre Société de Gynécologie et d'Obstétrique de la Suisse Romande - 70 Jahre Gynäkologische Gesellschaft der deutschen Schweiz
}

Von Heinrich Stamm

1896 Die Genfer Gynäkologen (im ganzen nur einige wenige) vereinigen sich, um 1896 den 2.internationalen Gynäkologenkongre $\beta$ in Genf zu organisieren. (Der nächste internationale Gynäkologenkongreß fand 1898 in St. Petersburg statt.)

Prof.O.Beuttner, 1907-1929 Ordinarius für Gynäkologie und Geburtshilfe in Genf, versucht die Gründung einer Schweizerischen Gynäkologengesellschaft. Von den angefragten Gynäkologen antworten 14, die Hälfte mehr oder weniger abschlägig. Die Antworten sind in der Frühjahrsausgabe der Gynaecologia Helvetica 1911 publiziert.

1901 Beuttner gibt die Gynaecologia Helvetica heraus (Tab.1) und legt damit den politischen Grundstein zur Entstehung gynäkologischer Fachgesellschaften in der Schweiz. Im gleichen Jahr wird die Foederatio Medicorum Helveticorum (FMH) gegründet.

1905 Die Welschschweizer Abonnenten der Gynaecologia Helvetica gründen am 2. März 1905 die Société de Gynécologie et d'Obstétrique de la Suisse Romande. Ähnliche Bestrebungen in der alemannischen Schweiz mißlangen, weil alle drei Lehrstuhlinhaber (Prof. O. von Herff in Basel, Prof. P. Müller in Bern und Prof.Th. Wyder in Zürich) (Tab.2) den Anschluß der deutschschweizerischen Gynäkologen an die Deutsche Gesellschaft für Gynäkologie und Geburtshilfe anstrebten und eine schweizerische Gesellschaft ablehnten. Das veranlaßte Prof. H. Guggisberg, 1911-1950 Ordinarius für Gynäkologie und Geburtshilfe in Bern, zu seinem berühmten Wort: «Der Staat tut gut daran, seine Kliniker dem eigenen Volke zu entnehmen.»

1911 Beuttner wirbt in einem Aufsatz: «Ist die Gründung einer deutschschweizerischen und einer gesamtschweizerischen gynäkologischen Gesellschaft möglich und wünschenswert?» für die gesellschaftliche Organisation 
aller Schweizer Gynäkologen. Er hat Erfolg. Am 10. Dezember treffen sich in Olten die Herren Prof.Beuttner (Genf), Dr.Debrunner (Frauenfeld), Dr. Dick (Bern), Dr. von Fellenberg (Bern), Dr. Jung (St. Gallen), Dr. Pfähler (Solothurn), Dr. Scheurer (Biel), Dr. Steiger (Bern), Dr. Streit (Bern) und Dr. Wormser (Basel) und beschließen die Gründung der gynäkologischen Gesellschaft der deutschen Schweiz. Geistiger Vater der Gründung ist Beuttner; Promotor, Organisator und Präsident der konstituierenden Sitzung in Olten ist von Fellenberg. In der Frühjahrssitzung 1912 im Berner Frauenspital werden die Statuten genehmigt, und Guggisberg wird zum ersten Präsidenten gewählt (Tab. 3).

1913 Seit dem Bestehen zweier Gynäkologengesellschaften macht sich beidseits der Wunsch nach gemeinsamen Kontakten geltend. Schon im Frühjahr 1913 findet die erste gemeinsame Sitzung in Genf statt. Thema: Therapie des septischen Aborts. Im Jahre der schweizerischen Landesausstellung 1914 treffen sich die Mitglieder der welschen und der deutschschweizerischen Gesellschaft ein zweites Mal in der Landesausstellung in Bern. Hauptthemen: 1.Tag: Strahlentherapie. 2.Tag: Sellheimsche Operationen.

1915 Dann kommt der Erste Weltkrieg, und der fatale Graben zwischen Welsch und Alemannisch verhindert weitere Kontakte. Das veranlaßt Dr.P.Jung, 1911-1940 Chefarzt der Frauenklinik St.Gallen, zu einer persönlichen schriftlichen Aufforderung an jedes einzelne der 25 Mitglieder der Société de la Suisse Romande zum engeren Zusammenschluß. Jung findet wider aller Prophezeiungen 22 bedingungslose Zustimmungen. Die Mitglieder der alemannischen Schweiz stehen geschlossen hinter ihm. So wird anläßlich der gemeinsamen Jahresversammlung 1915 in St. Gallen der Zusammenschlu $\beta$ der beiden unabhängigen Gynäkologengesellschaften der Schweiz in einer gemeinsamen Spitze mit Präsident und Sekretär beschlossen (Tab.4). Die Société Romande und die deutschschweizerische Gesellschaft bleiben unabhängig. Der gemeinsame Vorstand organisiert als verbindendes Element jährlich im Sommer die gemeinsame Jahrestagung. Daneben treffen sich die Mitglieder der Société Romande 3-4mal jährlich in Genf, Lausanne, Neuenburg, Fribourg und Wallis. Die Versammlungen der deutschschweizerischen Gynäkologen finden seit 1914 einmal jährlich im Mai im Tagsatzungssaal Baden statt, und zum gemeinsamen Mittagessen im Kursaal stiftet der Stadtrat regelmäßig Ehrenwein und Chräbeli. 
1916 Am 19./20. September 1916 findet die erste Versammlung unter gemeinsamer Leitung in der Maternité Lausanne statt (Tab.5). Erstes Spitzengremium der beiden Gesellschaften sind Dr.E. Wormser, Basel, Präsident, und Dr. R. von Fellenberg, Bern, Sekretär (Tab.4). Im Ausland bleibt die Vereinigung nicht unbemerkt. Die Münchener Medizinische Wochenschrift eröffnet eine scharfe Polemik, wogegen sich der neu ernannte gemeinsame Vorstand energisch zur Wehr setzt.

1918 Im Jahre 1911 wird Prof. H. Guggisberg als Nachfolger Prof. E. Kehrers auf den Berner Lehrstuhl berufen. 1916 folgt in Basel Prof. A. Labhardt seinem Vorgänger, Prof. von Herff. Damit sind zwei harte Gegner einer schweizerischen Gynäkologenorganisation durch zwei warme Befürworter ersetzt. Die universitäre Unterstützung der Gesellschaft erweist sich als besonders wichtig, als im Oktober 1918 anläßlich der Jahresversammlung in Genf eine Stellungnahme zum geplanten Abort-Artikel im schweizerischen Strafgesetzbuch nötig wird. P. Jung, St. Gallen, berichtet in Helvetica Medica Acta 6 (1937), S. 896, über diese Sitzung: «Alfred Gautier, der hochangesehene Genfer Strafrechtslehrer, hatte das juristische Referat übernommen. Während unsere Gesellschaft sich einstimmig gegen eine besondere Bestimmung über den therapeutischen Abort im Strafgesetzbuch aussprach und diesen - wie bisher in den meisten Kantonen - nur der Jurisdiction des ärztlichen Gewissens unterstellen wollte, verlangte der Jurist, daß darüber legiferiert werde, und befürwortete den Paragraphen des Entwurfs, der allerdings damals nur eine ganz allgemeine und sehr milde Fassung bot.»

1920 Die finanziellen Opfer, die Beuttner für seine in Genf erscheinende Gynaecologia Helvetica bringen muß, werden untragbar. Weder eine Obligatorischerklärung des Abonnements für alle Mitglieder noch eine Erhöhung des Mitgliederbeitrags auf 20 Franken (wovon 14 für die Zeitschrift) konnte das Eingehen des nun 20jährigen Gesellschaftsorgans verhindern. Nach längeren Verhandlungen mit der Wochenschrift und mit der Rundschau und mit der Chirurgengesellschaft kann schließlich in der Schweizerischen Medizinischen Wochenschrift ein neues Publikationsorgan gefunden werden (Tab. 1).

1928 werden die Versammlungen der deutschschweizerischen Gesellschaft von Baden nach Aarau in die Klinik von Dr. P. Hüssy, 1920-1947 Chefarzt der Frauenklinik Aarau, verlegt. Es institutionalisiert sich nach und nach 
eine regelmäßige Herbstversammlung (neben der gemeinsamen Sommertagung mit der Société Romande), an deren Organisation sich allmählich auch die Universitätskliniken von Zürich, Basel und Bern beteiligen.

1931 Im Rahmen der Foederatio Medicorum Helveticorum (FMH) wird die erste Spezialarztordnung geschaffen. Es gibt einen Spezialarzt für Geburtshilfe und einen für Gynäkologie.

1938 Deutschland ist die führende Nation in der medizinischen Welt. Die deutsche Sprache hat in der Naturwissenschaft den Stellenwert, den heute das Englische einnimmt. Damals existiert «der Deutsche Gynäkologenkalender», in dem alle Gynäkologen des großdeutschen Raumes verzeichnet werden. Bei der Neuauflage 1938 müssen sich nun sämtliche Gynäkologen, die im Kalender verzeichnet sein wollen, unterschriftlich zur deutschen Abstammung und zu den Nürnberger Rassengesetzen bekennen. Als dieses Ansinnen ruchbar wird, bricht unter den deutschschweizerischen Gynäkologen eine Welle der Entrüstung aus. Der Gesellschaftsvorstand ermächtigt den angesehensten Schweizer Fachvertreter, Prof.Guggisberg, Bern, zu Verhandlungen mit Prof. Stöckel, Ordinarius für Gynäkologie und Geburtshilfe in Berlin, dem Präsidenten der deutschen Gesellschaft für Gynäkologie und Geburtshilfe und Herausgeber des Gynäkologenkalenders. In einem Briefwechsel (den Dr.Paul Ehrler, Bern, im Bund vom 7.Januar 1978 publiziert hat) wird erreicht, daß die Schweizer Gynäkologen ihr unterschriftliches Bekenntnis zum Nazireich nicht abzulegen brauchten, daß sie aber Gewähr bieten sollten, daß keine Juden ins Deutsche Gynäkologenverzeichnis eingeschleust würden. Anläßlich der ordentlichen Herbstversammlung 1938 in Basel wird einstimmig beschlossen, daß die Schweizer Gynäkologen eine Aufnahme in den Deutschen Gynäkologenkalender ablehnen. Wenig später beginnt der selbstmörderische Zweite Weltkrieg, und die Beziehungen zwischen Schweizer und deutschen Gynäkologen werden aus ideologischen Gründen unmöglich.

1939 Totalrevision der Spezialarztordnung: Es wird die heutige Spezialarztauszeichnung FMH für Gynäkologie und Geburtshilfe geschaffen.

1946 Prof.Th. Koller, 1949-1969 Ordinarius für Gynäkologie und Geburtshilfe in Basel, erneuert in seiner Eigenschaft als Präsident der gynäkologischen Gesellschaft der deutschen Schweiz die 1938 eingestellten Bezie- 
hungen zu den Gynäkologen Deutschlands. Insbesondere organisiert er gemeinsam mit Prof.August Mayer, Tübingen, und Prof.Burger, Straßburg, die Oberrheinische Gesellschaft für Geburtshilfe und Gynäkologie in ihrer ersten Nachkriegssitzung 1946 in Basel neu. Aus verständlichen politischen Gründen kann die offizielle Gründung der französischen Sektion erst 1973 erfolgen. Es sei in Erinnerung gerufen, daß die Oberrheinische Gesellschaft 1905, als das Elsaß noch deutsch war, von Prof.Hegar, Freiburg, und Prof. Fehling, Basel und Straßburg, gegründet wurde und daß sie nach dem Auseinanderbrechen im Ersten Weltkrieg von Prof.Alfred Labhardt, 1916-1942 Ordinarius für Gynäkologie und Geburtshilfe in Basel, in den Zwischenkriegsjahren, wenigstens zwischen der Schweiz und Deutschland, wieder aufgebaut werden konnte. Schon $1933 \mathrm{kam}$ es indessen erneut zu unüberbrückbaren Spannungen. Aus einem Brief von Dozent Pfleiderer, Stuttgart, von 1934 geht hervor, daß nicht nur die Gesellschaft von volksfremden Elementen, sondern auch der Vorstand von unerwünschten Schweizern «gereinigt» werden sollte.

1954 organisiert Prof. Hubert de Watteville, 1946-1976 Ordinarius für Gynäkologie und Geburtshilfe in Genf, den Weltkongreß für Gynäkologie in Genf. Zu diesem Anlaß wird auf Initiative de Wattevilles die Fédération Internationale de Gynécologie et d'Obstétrique (FIGO) gegründet. Prof.de Watteville ist erster Präsident, dann bis 1976 Generalsekretär. Unter seiner Leitung wächst die FIGO zu einer weltumspannenden und mächtigen Organisation an. Ihr gehören heute 83 nationale Gynäkologengesellschaften mit über 30000 Mitgliedern an.

1964 gründet Koller zusammen mit den deutschschweizerischen Gynäkologen die Schweizerische Gesellschaft für Familienplanung. Er ist erster Präsident; erste Sekretärin ist Frau Prof. Marianne Mall, Basel.

Die Stimmen zur Vereinigung der beiden schweizerischen Gynäkologengesellschaften verstummen nicht. Mit der Wahl von Dr.H.J. Wespi, 1947-1974 Chefarzt der Frauenklinik Aarau, zum Sekretär der beiden Schweizer Gynäkologengesellschaften kommt im Jahre 1958 ein Mann an die Spitze der gesamtschweizerischen Gynäkologen, der mit großer Energie die Vorarbeiten zur Vereinigung an die Hand nimmt. In unermüdlicher fünfjähriger Sekretariatstätigkeit (1958-1962) schließt er zusammen mit den Gesellschaftspräsidenten, Prof.H.de Watteville, Genf, Prof. O. Käser, 
St.Gallen, Prof.P.Dubuis, Lausanne, Prof.G.Müller, Bern, und Dr. P.Berthoud, Neuchâtel, die Arbeiten so weit ab, daß 1964 Dr.P.Bloch, Lausanne, als Gemeinschaftspräsident der Société de Gynécologie et d'Obstétrique de la Suisse Romande und der gynäkologischen Gesellschaft der deutschen Schweiz, die beiden Gesellschaften auflösen und in eine einzige Schweizerische Gesellschaft für Gynäkologie überführen kann (Tab.6). Die bisherige Société de la Suisse Romande existiert seit 1964 als Groupement Romand de la Société Suisse de Gynécologie weiter. Nachträglich bilden sich in der deutschen Schweiz mehrere lokale Sektionen, z. B. die Gynaecoba in Basel 1959, die Gynäkologenvereinigung des Kantons Zürich 1966, die Gynäkologenvereinigung Aargau 1970, der Verein der freipraktizierenden Gynäkologen des Kantons St. Gallen 1976, das Gynäkologenkollegium der Stadt Bern 1977 und die freie Vereinigung der Gynäkologen der Zentralschweiz 1979.

1965 Die Schweizerische Gesellschaft für Gynäkologie erhält unter ihrem ersten Präsidenten, Prof.E.Held, 1950-1971 Ordinarius für Gynäkologie und Geburtshilfe in Zürich, und ihrem ersten Sekretär, Dr.A. Reist, 1926-1963 Chefarzt der Pflegerinnenschule Zürich, ihre Statuten, die heute noch in Kraft sind.

1966 Nach der Vereinigung der beiden schweizerischen Gynäkologengesellschaften zur Schweizerischen Gesellschaft für Gynäkologie fallen, im Gegensatz zur Gepflogenheit in der welschen Schweiz, die deutschschweizerischen Herbsttagungen dahin. Unermüdlich arbeitet der damalige Chefarzt der Sanitasklinik in Zürich, Dr. Alfred Bucher-von Grüningen, der sich seit Jahren für die Gynäkologenfortbildung einsetzt, an einer regelmäßig stattfindenden Fortbildungstagung. Im März 1968 kann der erste Herbstfortbildungskurs in Genf stattfinden. Dann wird er 1970 in Basel, 1971, 1972 und 1973 in Baden, 1974 in Zürich, 1975 in Basel und 1976 in Liestal abgehalten. Daneben ist der seit 1966 von Prof. R. Wenner organisierte Fortbildungskurs in gynäkologischer Endokrinologie und Familienplanung als fester Bestandteil der schweizerischen Gynäkologenausbildung etabliert. 1977 übernimmt Prof. Otto Käser, seit 1969 Ordinarius für Gynäkologie und Geburtshilfe in Basel, die Organisation der Herbstfortbildungskurse. Als Basler Fortbildungskurs für praktische Ärzte und Gynäkologen wird seither der von Bucher-von Grüningen geschaffene Herbstfortbildungskurs weitergeführt. 
1967 Mit der Präsidialzeit von Prof. W. R. Merz, 1957-1980 Ordinarius für Gynäkologie und Geburtshilfe in Lausanne, wird das Schwergewicht der Vorstandstätigkeit der Schweizerischen Gesellschaft für Gynäkologie auf die Verbesserung der Gynäkologenausbildung gelegt. Die Programmpunkte sind: - Schaffung einer Ausbildungskommission, Collège des Gynécologues genannt, deren Präsident Prof. Merz heute noch ist - Obligatorische chirurgische Ausbildung im Rahmen der gynäkologischen Spezialarztausbildung - Verlängerung der Spezialarztausbildung von 5 Jahren auf 6 Jahre - Normierung der Spezialarztausbildung durch ein Cahier de stages Einführung eines Spezialarztexamens - Verbesserung der Ausbildung zukünftiger gynäkologischer Strahlentherapeuten - Verbesserung der Ausbildung zukünftiger gynäkologischer Chefärzte.

Jahrelang ist Prof. Merz zu Besprechungen mit dem Zentralvorstand der Verbindung der Schweizer Ärzte (FMH), die der Bundesrat mit der Spezialarztausbildung betraut hat, nach Bern gereist. Er ist auf taube Ohren gestoßen. Lediglich das Eidgenössische Amt für Strahlenschutz organisiert seit 1968 einen obligatorischen Kurs in Strahlenschutz für gynäkologische Strahlentherapeuten.

1969 Die Firma Schering, Schweiz, stiftet den mit 10000 Franken dotierten Schering-Preis. Er wird alle zwei Jahre im Rahmen einer feierlichen Zeremonie an der Jahresversammlung «zur Prämierung wertvoller Arbeiten auf dem Gebiete der gynäkologischen und geburtshilflichen Endokrinologie» ausgeschüttet. Bisherige Preisträger:

1969 in Gstaad: Prof. Dr. med. Paul Keller, Zürich

1971 in Crans: keine Arbeit eingereicht

1973 in Basel: $\quad$ Dr. med. Ulrich Gigon, Bern

1975 in Nyon: $\quad$ 1) Dres. med. K. B. Ruf, H. J. Kilchen und M. Wilkinson, Genève

2 a) Dr. chem. P. R. Huber, Basel, und Dr.med. J. Frech, Zürich

2 b) Dr.med.H.G.Kopp, Zürich

1977 in Lugano: Dres. med. K. Stalder und R. Haldemann, Bern

1979 in Montreux: Dres. med. Irmi Rey-Stocker, Frau M.M.Zufferey, Frau M. Th. Lemarchaud und Frau M. Rais, Lausanne

1981 in Genf: $\quad$ keine Arbeit eingereicht

1969 wird auf Grund eines Beschlusses an der Jahresversammlung in Basel 
die Spezialarztausbildung mit einem Cahier de stages normiert. Dieses Cahier galt lange Zeit als vorbildlich für die schweizerische Spezialarztausbildung. Es wird zur Zeit revidiert.

1972 Frau Dr. Irmi Rey-Stocker gründet auf Anregung des Vorstandes der Schweizerischen Gesellschaft für Gynäkologie die Schweizerische Arbeitsgruppe für Kinder- und Adoleszentengynäkologie. Frau Dr. Rey ist seit 1971 Generalsekretärin der Fédération Internationale de Gynécologie Infantile et Juvénile (F.I.G.I.J.) und seit 1965 Chefarzt der geburtshilflich-gynäkologischen Abteilung des Hôpital d'Arrondissement de Sierre/Wallis.

1976 legt Prof. W. Herrmann, seit 1976 Ordinarius für Gynäkologie und Geburtshilfe in Genf, die Unterlagen für ein Spezialarztexamen vor. Es verdient erwähnt zu werden, daß der Zentralvorstand der Verbindung der Schweizer Ärzte (FMH) zur Zeit ebenfalls Anstrengungen unternimmt, um die Spezialarztausbildung zu verbessern, und daß sich die derzeitigen Pläne nicht allzusehr von den Ideen von Prof. Merz und Prof. Herrmann unterscheiden.

1977 Prof. de Watteville gründet in Zusammenarbeit mit den Spitzen der Schweizerischen Gesellschaft für Gynäkologie die International Association of Maternal and Neonatal Health (IAMANEH). De Watteville wird erster Generalsekretär. Das Generalsekretariat hat Sitz in Genf. Heute umfaßt die IAMANEH 25 nationale Sektionen.

Dank der Initiative und mehrjährigen Vorarbeit von Prof. Max Berger, seit 1967 Ordinarius für Gynäkologie und Geburtshilfe in Bern, kann im Rahmen einer Versammlung in Lugano die Schweizerische Gesellschaft für Senologie gegründet werden. Die Gynäkologengesellschaft gibt ihrer jungen Tochter ein Startkapital von Fr.20000.- mit. Erster Präsident ist Dr. A. Gallino, seit 1957 Chefarzt am Kantonsspital San Giovanni in Bellinzona.

1979 Prof. H. Bossart, seit 1980 Ordinarius für Gynäkologie und Geburtshilfe in Lausanne, gründet mit finanzieller Hilfe der Firma Schering, Schweiz, das Informationsbulletin der Schweizerischen Gesellschaft für Gynäkologie. Damit besitzt die Schweizerische Gynäkologengesellschaft zum erstenmal in ihrer Geschichte ein eigenes standespolitisches Informationsorgan. 
1980 Der Vorstand der Gynäkologengesellschaft beschließt eine bessere Betreuung der Chefärzte. PD Dr. E. Hochuli, seit 1963 Chefarzt am Kantonsspital Münsterlingen, wird als Delegierter in der Konsultativkommission der Verbindung der Schweizer Ärzte für Chefarztfragen und als Präsident der gynäkologischen Chefarztkonferenz beauftragt, jährliche Fortbildungskurse für Chefärzte zu organisieren. Der erste Kurs findet 1980 in Freiburg im Breisgau, der zweite 1981 in Münsterlingen, Thurgau, statt.

1981 Jubiläumsfeier «75 Jahre Société Romande, 70 Jahre Gesellschaft der deutschen Schweiz» anläßlich des Ehrenessens, das seit 1977 auf Anregung des damaligen Präsidenten, Dr.A.Gallino, alle zwei Jahre im Vorstand seinen Ehrenmitgliedern offeriert wird.

Tab.1: Verzeichnis der offiziellen Gesellschaftszeitschriften der Schweizerischen Gynäkologengesellschaften

1901-1920 Gynaecologia Helvetica. Chefredaktor O.Beuttner, Genf. Verlag Kundis, Genf. $1920 \mathrm{mu} ß$ das Erscheinen aus finanziellen Gründen eingestellt werden.

1921-1934 Schweizerische Medizinische Wochenschrift. Verlag Schwabe, Basel.

1934-1939 Helvetica medica acta. Verlag Schwabe, Basel.

1939-1946 Monatsschrift für Geburtshilfe und Gynäkologie. Chefredaktoren: E.Andres, Zürich, und Th. Koller, Zürich und Basel. Verlag Karger, Basel. Seit 1939 (Vol. 109) offizielles Publikationsorgan der gynäkologischen Gesellschaft der deutschen Schweiz.

1946-1969 1946 erhält Vol.122 der Monatsschrift als offizielles Publikationsorgan aller gynäkologischen Gesellschaften der deutschen Schweiz den Namen Gynaecologia. Chefredaktoren: E. Andres, Zürich, und Th.Koller, Basel. Verlag Karger, Basel. Ihr Erscheinen muß 1969 aus finanziellen Gründen eingestellt werden (Vol.168). Damalige Redaktion: P.Bloch, Lausanne, Th.Koller, Basel, und O. Käser, Frankfurt.

1970-1972 Schweiz. Zeitschrift für Gynäkologie und Geburtshilfe - Revue Suisse de Gynécologie et d'Obstétrique. Redaktoren: W.R.Merz, Lausanne, M.Berger, Bern, und W.Schreiner, Zürich. Verlag Schwabe, Basel. Wiederum sind es finanzielle Gründe, die eine Neudisposition nötig machen.

Seit 1973 Gynäkologische Rundschau. Redaktoren: M.Berger, Bern, H.Bossart, Lausanne, H.Hußlein, Wien, H.Schwalm, Hallgarten, und R.Wenner, Liestal. Verlag Karger, Basel. 
Tab.2: Die Lehrstuhlinhaber für Gynäkologie und Geburtshilfe an den schweizerischen 3Universitäten

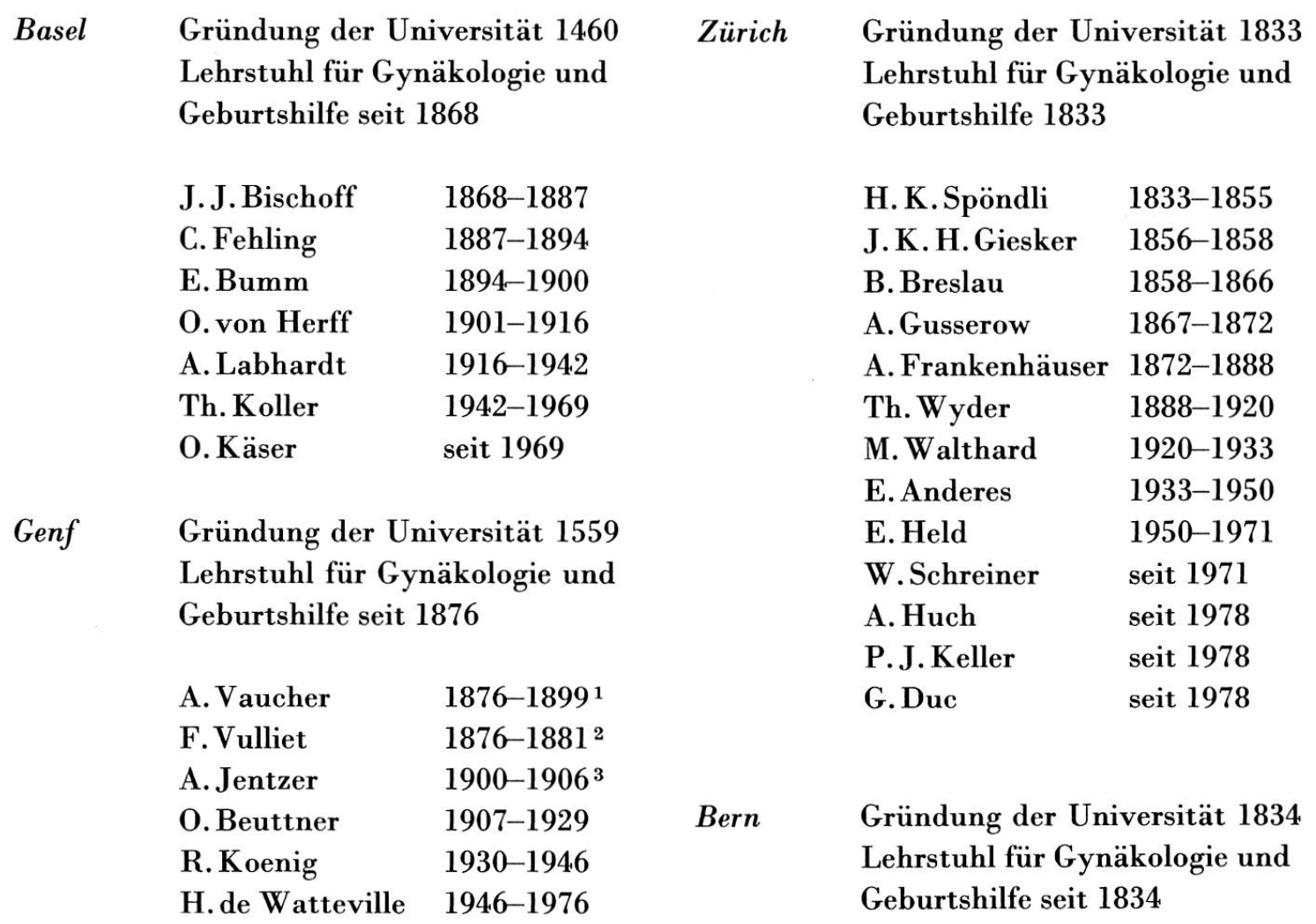

$\begin{array}{ll}\text { J. J.Hermann } & 1834-1861 \\ \text { Th. Hermann } & 1861-1867 \\ \text { A. Breisky } & 1867-1873 \\ \text { P.Müller } & 1873-1910 \\ \text { E. Kehrer } & 1910-1911 \\ \text { H.Guggisberg } & 1911-1950 \\ \text { W. Neuweiler } & 1950-1966 \\ \text { M. Berger } & \text { seit } 1967\end{array}$

Lausanne Gründung der Universität 1890 Lehrstuhl für Gynäkologie und Geburtshilfe seit 1890

\footnotetext{
${ }^{1}$ Geburtshilfe

${ }^{2}$ Gynäkologie

${ }^{3}$ Geb. + Gyn.
}
C. Roux
G. Rossier
R. Rochat
W. R.Merz
H. Bossart 
Tab.3: Präsidenten und Sekretäre der Gynäkologischen Gesellschaft der deutschen Schweiz seit der Gründung im Jahre 1911 bis zur Liquidation 1964

\begin{tabular}{|c|c|}
\hline Jahr & nten \\
\hline 1912-1914 & Prof. H. Guggisberg, Bern \\
\hline 1915-1916 & Dr. E. Wormser, Basel \\
\hline 1917-1918 & Dr. R.von Fellenberg, Bern \\
\hline $1919-1920$ & Dr.P. Jung, St. Gallen \\
\hline 1921-1922 & Prof. W.Goenner, Basel \\
\hline 1923-1924 & Prof. H. Meyer-Rüegg, Zürich \\
\hline 1925-1926 & Prof. A. Labhardt, Basel \\
\hline $1927-1928$ & Prof.M. Walthard, Zürich \\
\hline $1929-1930$ & Dr. W. Bigler, St. Gallen \\
\hline 1931-1932 & Dr.P.Hüssy, Aarau \\
\hline 1933-1934 & Prof. E.Anderes, Zürich \\
\hline 1935-1936 & Prof. E. Frey-Bolli, Zürich \\
\hline $1937-1938$ & Prof. H.Schultheiß, Basel \\
\hline $1939-1940$ & Dr. A. Reist, Zürich \\
\hline $1941-1942$ & Prof. W. Neuweiler, Bern \\
\hline $1943-1944$ & Dr.C. Brunner, Winterthur \\
\hline $1945-1946$ & Prof.Th. Koller, Basel \\
\hline $1947-1948$ & Prof. Fr. Ludwig, Bern \\
\hline $1949-1950$ & Prof. R. Wenner, Basel \\
\hline $1951-1952$ & Prof.E. Held, Zürich \\
\hline $1953-1954$ & Dr. A. Bucher, Zürich \\
\hline $1955-1956$ & Dr.H.J. Wespi, Aarau \\
\hline $1957-1958$ & Dr. A.Scharplatz, Chur \\
\hline $1959-1960$ & Prof. O. Käser, St. Gallen \\
\hline 1961-1962 & Prof. C. Müller, Bern \\
\hline 1963-1964 & Prof.E.Glatthaar, W \\
\hline
\end{tabular}

\author{
Sekretäre \\ Dr. E. Wormser, Basel \\ Dr. R.von Fellenberg, Bern \\ Dr.P.Scheurer, Biel \\ Prof. H. Meyer-Rüegg, Zürich \\ Prof. H. Meyer-Rüegg, Zürich \\ Prof. M. Walthard, Zürich \\ Dr.P.Hüssy, Aarau \\ Dr.P.Hüssy, Aarau \\ Dr.P.Hüssy, Aarau \\ Prof. H. Schultheiß, Basel \\ Prof. H. Schultheiß, Basel \\ Prof. H. Schultheiß, Basel \\ Dr. A. Gengenbach, Basel \\ Dr. A. Gengenbach, Basel \\ Prof.Th. Koller, Zürich \\ Prof. R. Wenner, Basel \\ Prof. R. Wenner, Basel \\ Prof.E. Held, St. Gallen \\ Dr. A. Bucher, Zürich \\ Dr. H. J. Wespi, Aarau \\ Dr. A. Scharplatz, Chur \\ Prof. O. Käser, St. Gallen \\ Prof. C. Müller, Bern \\ Prof.E.Glatthaar, Winterthur \\ Dr. M. Etterich, Basel \\ Dr. G. Rieben, Interlaken
}

Tab.4: Präsidenten und Sekretäre des 1916 unter dem Namen «Schweizerische Gesellschaft für Gynäkologie - Société Suisse de Gynécologie» konstituierten Spitzenorgans der gynäkologischen Gesellschaft der deutschen Schweiz und der Société de Gynécologie et d'Obstétrique de la Suisse Romande bis zur endgültigen Fusion im Jahre 1964.

Dem Präsidium oblag unter anderem die Organisation der Jahresversammlungen bis 1964. 


\section{Präsidenten}

Dr.E. Wormser, Basel

Prof. O. Beuttner, Genève

Dr. P. Jung, St. Gallen

Dr. P. Chapuis, Genève

Prof. W. Goenner, Basel

Dr. H. Chassot, Genève Prof. H. Meyer-Rüegg, Zürich

Gemeinschaftstagung mit der Prof. C. Henschen, St. Gallen

Prof. A. Labhardt, Basel

Dr. R. Jaeggy

Prof. M. Walthard, Zürich

Dr. F. Chatillon, Genève

Dr. W. Bigler, St. Gallen

Prof.E.Anderes, Zürich

Dr. H. Henneberg, Genève

Dr.P.Hüssy, Aarau

Prof. E.Anderes, Zürich

Prof. R. Rochat, Lausanne

Prof.E.Frey, Zürich

Dr. H.F. Decoppet, Lausanne

Dr.E.Gueissaz, Neuchâtel

Prof. H. Schultheiß, Basel

Dr. A. Reist, Zürich

Prof. M. Muret, Lausanne

Prof. W. Neuweiler, Bern

Dr. F. Chatillon, Genève

Dr.C.Brunner, Winterthur

Dr. E. de Meuron, Lausanne

Prof.Th.Koller, Basel

Dr. Ch. Waegeli, Genève

Prof. F. Ludwig, Bern

Dr. E.Gueissaz, Neuchâtel

Prof. W. Wenner, Basel

Prof. R. Rochat, Lausanne

Dr. R. Meylan, Genève

Prof.E. Held, Zürich

Dr. A. Bucher, Zürich

Prof. R. Rochat, Lausanne

Dr. H. J. Wespi, Aarau

Prof. W. Geisendorf, Genève

Dr. A. Scharplatz, Chur

Prof.H.de Watteville, Genève

Prof. O. Käser, St. Gallen

Prof. P. Dubuis, Lausanne

Prof. C. Müller, Bern

Dr.P. Berthoud, Neuchâtel

Prof.E.Glatthaar, Winterthur

Dr. P. Bloch, Lausanne

\section{Sekretäre}

Dr. R.von Fellenberg, Bern

Dr. H. Chassot, Genève

Prof. H. Meyer-Rüegg, Zürich

Dr. R. Martin, Genève

Prof. H. Meyer-Rüegg, Zürich

Dr. F. Chatillon, Genève

Prof. M. Walthard, Zürich

Gesellschaft für Chirurgie

Prof. E. Monnier, Zürich

Dr.P.Hüssy, Aarau

Dr. E. Gueissaz, Neuchâtel

Dr.P.Hüssy, Aarau

Prof. R. Rochat, Lausanne

Dr.P.Hüssy, Aarau

Dr. P. Hüssy, Aarau

Dr. F.Chatillon, Genève

Prof. H. Schultheiß, Basel

Prof. H. Schultheiß, Basel

Dr. F. Chatillon, Genève

Prof. H. Schultheiß, Basel

Prof. H. Schultheiß, Basel

Prof.E. Frey, Zürich

Prof.E. Frey, Zürich

Dr.E.Gueissaz, Neuchâtel

Dr. E.Gueissaz, Neuchâtel

Dr. E. Gueissaz, Neuchâtel

Dr. E.Gueissaz, Neuchâtel

Dr. P. Jung, St. Gallen

Dr. P. Jung, St. Gallen

Dr. P. Jung, St. Gallen

Dr.P. Jung, St. Gallen

Dr. P. Jung, St. Gallen

Dr. P. Jung, St. Gallen

Prof. W. Geisendorf, Genève

Prof. W. Geisendorf, Genève

Prof. W. Geisendorf, Genève

Prof. R. Wenner, Basel

Prof. R. Wenner, Basel

Prof. W. Wenner, Basel

Dr. R. Meylan, Genève

Dr. R. Meylan, Genève

Dr. R. Meylan, Genève

Dr. H. J. Wespi, Aarau

Dr. H. J. Wespi, Aarau

Dr. H. J. Wespi, Aarau

Dr. H. J. Wespi, Aarau

Dr. H. J. Wespi, Aarau

Dr.P.Berthoud, Neuchâtel

Dr.P.Berthoud, Neuchâtel 
Tab.5: Die Orte der Jahresversammlungen der vereinigten Schweizerischen Gesellschaft für Gynäkologie 1916-1981

\author{
1916 1. Versammlung Lausanne \\ 1917 2. Versammlung Basel \\ 1918 3. Versammlung Genf \\ 1919 4. Versammlung Bern \\ 1920 5. Versammlung Fribourg \\ 1921 6. Versammlung Zürich \\ 19227 .Versammlung Lausanne \\ 1923 keine Jahresversammlung
}

1924

\section{Versammlung Basel}

1925 9. Versammlung St. Gallen

1926 10. Versammlung Neuchâtel

1927 11. Versammlung Bern

1928 12. Versammlung Lugano

1929 13. Versammlung Basel

1930 14. Versammlung Zürich

1931 15. Versammlung Genf

1932 16. Versammlung Bern

1933 17. Versammlung Luzern

1934 18. Versammlung Lausanne

1935 19. Versammlung Zürich

1936 20. Versammlung Aarau

1937 21. Versammlung Montana

1938 22. Versammlung Basel

1939 23. Versammlung Zürich

1940 keine Jahresversammlung

1941 24. Versammlung Bern

1942 25. Versammlung Neuchâtel

1943 26. Versammlung Luzern

1944 27. Versammlung St. Gallen

194528 . Versammlung Lugano

1946 29. Versammlung Basel

1947 30. Versammlung Bern

1948 31. Versammlung Genf

194932. Versammlung Zürich

195033. Versammlung Interlaken

1951 34. Versammlung Lausanne

1952 35. Versammlung Bürgenstock
19./20.9. Maternité

13./14.10. Frauenklinik

5./6.10. Amphithéâtre de la Maternité

8./9.10. Frauenklinik

2./3.10. Amphithéâtre de l'Institut Pathologique

15./16.10. Frauenklinik

23./24.9. Maternité

17./19.8. Congrès de la Fédération des Sociétés de Gynécologie et d'Obstétrique de langue française à Genève

21./22.6. Bürgerspital, gemeinsam mit der Schweiz. Gesellschaft für Chirurgie

24./25.10. Aula der Handelshochschule

25./26.9. Universität

29./30.10. Frauenklinik

20./21.10. Liceo cantonale (1.Sitzung im Tessin)

20./21.4. «Schweizer Gynäkologentag»

3./4.5. Auditorium Frauenklinik

9./10.5. Grand Amphithéâtre de la Maternité

30.4./1.5. Frauenklinik

24./25.6. Verwaltungsratssaal der SUVA

9./10.6. Grand Amphithéâtre de la Maternité

7./8.7. Hörsaal der Universitäts-Frauenklinik

9./10.5. Kantonsspital und Schinznach-Bad

12./13.6. Golfhotel

18./19.6. Frauenklinik

24./25.6. Frauenklinik und Pflegerinnenschule

Aktivdienst

9.11. Frauenklinik

3./4.10. Bâtiment du laboratoire suisse de recherches horlogères

29./30.6. Sitzungssaal der SUVA

3./4.6. Frauenklinik des Kantonsspitals

26./27.5. Liceo cantonale

22./23.6. Frauenklinik der Universität

21./22.6. Frauenklinik der Universität

29./30.5. Athénée et Maternité

25./26.6. Universitäts-Frauenklinik

24./25.6. Theatersaal des Kursaals

26./27.5. Amphithéâtre de l'Institut d'Anatomie pathologique

21./22.6. Grand-Hotel 
1953

51. Versammlung Gstaad

53. Versammlung Crans-sur-

Sierre

197254 . Versammlung Locarno

1973 55. Versammlung Basel

1974 56. Versammlung Luzern

1975 57. Versammlung Nyon

1976 58. Versammlung Davos

1977 59. Versammlung Lugano

1978 60. Versammlung Biel

1979 61. Versammlung Montreux

1980

1981
62. Versammlung St. Gallen

18. -19.6
11.- -13.6

Cinéma du Parc

Congrès de la Fédération internationale de

Gynécologie et d'Obstétrique (FIGO) à Genève

11./12.6. Kurtheater

16./17.6. Liceo cantonale

22./23.6. Aula de l'Université

10./11.6. Großratssaal, gemeinsam mit der Schweiz. Gesellschaft für Urologie

10./11.5. Palace-Hotel

23./24.6. Auditorium de l'Institut d'Anatomie

15./16.6. Kursaal, Theatersaal

24./25.5. Hotel Elite

16./17.5 Salle des Remparts

6./7.6. Stadttheater, gemeinsam mit der Schweiz. Gesellschaft für Anästhesiologie

21.-23.5. Château de Porrentruy, Salle du Tribunal de district

10.-12.6. Aula magna del Liceo cantonale, gemeinsam mit der Schweiz. Gesellschaft für Pädiatrie

9.-11.6. Amphithéâtre de la Clinique universitaire de Pédiatrie

28.-30.6. Berufsschulhaus Kreuzackerquai und Berufsschulhaus des Kaufmännischen Vereins, gemeinsam mit der Schweiz. Gesellschaft für Familienplanung

27.-29.6. Palace-Hotel

19.-21.6. Vortragssaal Kantonsspital Winterthur

18.-20.6. Hôtel du Golf

22.-25.6. Palazzo dei Congressi

28.-30.6. Kongreßzentrum der Firma F. Hoffmann-La Roche

20.-22.6. Verkehrshaus

19.-21.6. Aula du Collège secondaire et auditoire secondaire de l'Hôpital de Nyon

1.-3.7. Kongreßhaus Davos-Platz

30.6.-2.7. Palazzo dei Congressi

22.-24.6. Kongreßhaus Biel

28.-30.6. Maison des Congrès, 27.-28.6. gemeinsam mit der Schweiz. Gesellschaft für Familienplanung

19.-21.6. Kongreßhaus Schützengarten, gemeinsam mit dem Schweiz. Hebammenverband Palais des Expositions 
Tab.6: Präsidenten und Tagungsorte der 1964 neu konstituierten Schweizerischen Gesellschaft für Gynäkologie

\begin{tabular}{|c|c|c|}
\hline Jahr & Präsidenten (Sekretäre) & Jahrestagungen (Organisation) \\
\hline \multirow[t]{2}{*}{ 1965-1966 } & Prof. E. Held, Zürich & 1965 Porrentruy (Dr.D.Stucki) \\
\hline & (Dr. A. Reist) & 1966 Lugano (Dr. C. Scacchi) \\
\hline \multirow[t]{2}{*}{$1967-1968$} & Prof. W. R. Merz, Lausanne & 1967 Genf (Prof. H. de Watteville) \\
\hline & (Dr. A. Reist) & 1968 Solothurn (Dr. A. Wacek) \\
\hline \multirow[t]{2}{*}{$1969-1970$} & Dr.G. Rieben, Interlaken & 1969 Gstaad (Dr. G. Rieben) \\
\hline & (Prof. H.Stamm) & 1970 Winterthur (Prof. E. Glatthaar) \\
\hline \multirow[t]{2}{*}{$1971-1972$} & Dr.J.P.d'Ernst, Genf & 1971 Crans-sur-Sierre (Irmi Rey-Stocker) \\
\hline & (Prof. H.Stamm) & 1972 Locarno (Dr. M. Rusca) \\
\hline \multirow[t]{2}{*}{$1973-1974$} & Prof. G. A. Hauser, Luzern & 1973 Basel (Prof. O. Käser) \\
\hline & (Prof.H.Stamm) & 1974 Luzern (Prof. A. Hauser) \\
\hline \multirow[t]{2}{*}{$1975-1976$} & Prof. M. Berger, Bern & 1975 Nyon (Dr.J.P.d'Ernst) \\
\hline & (Prof. H.Stamm) & 1976 Davos (Dr.Silvia Bono) \\
\hline \multirow[t]{2}{*}{$1977-1978$} & Dr.A.Gallino, Bellinzona & 1977 Lugano (Dr. G.P. Balmelli) \\
\hline & (Prof.H.Stamm) & 1978 Biel (Prof. U. Herrmann) \\
\hline \multirow[t]{3}{*}{$1979-1980$} & Prof. H. Bossart, Lausanne & 1979 Montreux (Prof. H. Bossart), \\
\hline & (Prof. H.Stamm) & $\begin{array}{l}\text { gemeinsam mit der Schweiz. Gesell- } \\
\text { schaft für Familienplanung } \\
\text { (Dr. C. Revaz) }\end{array}$ \\
\hline & & 1980 St. Gallen (Prof. U. Haller) \\
\hline $1981-1982$ & $\begin{array}{l}\text { Prof. W.Schreiner, Zürich } \\
\text { (Dr. H.-J. Welti) }\end{array}$ & 1981 Genf (Prof. W. Herrmann) \\
\hline
\end{tabular}

Tab.7: Mitgliederzahl und Jahresbeiträge der Gynäkologischen Gesellschaft der deutschen Schweiz, ab 1964 zusammen mit der Société de Gynécologie et d'Obstétrique de la Suisse Romande (Schweizerische Gesellschaft für Gynäkologie)

$\begin{array}{lcllll}\text { Jahr } & \text { Mitglieder } & \text { Jahresbeitrag } & \text { Jahr } & \text { Mitglieder } & \text { Jahresbeitrag } \\ 1912 & 15 & \text { Fr. 5.- } & 1949 & 157 & \text { Fr. 45.- } \\ 1915 & 19 & \text { Fr. 10.- } & 1959 & 197 & \text { Fr. 55.- } \\ 1918 & 56 & \text { Fr. 15.- } & 1964 & 213 & \text { Fr. 55.- } \\ 1920 & 76 & \text { Fr. 20.- } & 1965 & 250 & \text { Fr. 85.- } \\ 1925 & 88 & \text { Fr. 10.- } & 1967 & 289 & \text { Fr. 90.- } \\ 1933 & 104 & \text { Fr. 15.- } & 1975 & 492 & \text { Fr. 120.- } \\ 1937 & 138 & \text { Fr. 25.- } & 1980 & 583 & \text { Fr. 120.- } \\ 1947 & 152 & \text { Fr. 38.- } & & & \end{array}$




\section{Tab.8: Hauptthemata an den Jahrestagungen der Schweizerischen Gesell- schaft für Gynäkologie}

1913 Genf

1914 Bern

1915 St.Gallen

1916 Lausanne

1917 Basel

1918 Genève

1919 Bern

1920 Fribourg

1921 Zürich

1922 Lausanne

1924 Basel

1925 St.Gallen

1926 Neuchâtel

1927 Bern

1928 Lugano

1929 Basel

1930 Zürich

1931 Genève

1932 Bern

1933 Luzern

1934 Lausanne

1935 Zürich

1936 Aarau

1937 Montana

1938 Basel

1939 Zürich

1940

1941 Bern

1942 Neuchâtel
Erste gemeinsame Tagung der Société de Gynécologie et d'Obstétrique de la Suisse Romande und der Gynäkologischen Gesellschaft der deutschen Schweiz

Hauptthema: Therapie des septischen Aborts

Tagung anläßlich der Schweiz. Landesausstellung

1. Hauptthema: Strahlentherapie des gynäkologischen Karzinoms

2. Hauptthema: Sellheimsche Operation

Konstituierung des gemeinsamen Vorstandes der Société Romande und der deutschschweizerischen Gesellschaft

Kein Hauptthema

Wehenmittel

Beziehungen von Magen- und Darminfektionen zur Schwangerschaft und zu den Funktionen des weiblichen Genitalsystems

Der legale Abort und der schweizerische Strafgesetzentwurf

Funktionelle Uterusblutungen

Strahlenbehandlung beim Karzinom

Nierenerkrankungen in der Schwangerschaft

Die weibliche Gonorrhoe

Keine Jahresversammlung wegen des Kongresses der Ligue des Sociétés de Gynécologie de langue française in Genève

Thrombose und Embolie

Abortparagraph

Indikation, Technik und Resultate des Kaiserschnittes

Kein Hauptthema

Vorzeitiger Blasensprung

Die funktionellen Betriebsstörungen des weiblichen Genitalsystems

Therapie des weiblichen Genitalprolapses

Thema unbekannt

Virginelle Blutungen

1. Therapie des engen Beckens

2. Indikation und Technik des Kaiserschnitts

1. Pathogenese und Therapie des Fluor vaginalis

2. Eklampsie

Fluor albus, Pathogenese und Therapie

Kein Hauptthema

Geburtsleitung bei engem Becken

Kein Hauptthema

Fragen der Geburtenregelung

Keine Jahrestagung wegen Generalmobilmachung

Tuberkulose der Genitalorgane

Gonorrhoe-Therapie 


\begin{tabular}{|c|c|c|}
\hline 1943 & Luzern & Vitamine in der Gynäkologie und Geburtshilfe \\
\hline 1944 & St.Gallen & Die Sterilität und ihre Behandlung \\
\hline 1945 & Lugano & Moderne Hormontherapie \\
\hline 1946 & Basel & Es wurde beschlossen, nur jedes zweite Jahr ein Hauptthema zu behandeln. \\
\hline 1947 & Bern & Die Genital-Tuberkulose der Frau \\
\hline 1948 & Genève & Kein Hauptthema \\
\hline 1949 & Zürich & Mortalité prénatale \\
\hline 1950 & Interlaken & Kein Hauptthema \\
\hline 1951 & Lausanne & Die Schmerzbekämpfung in der Geburtshilfe \\
\hline 1952 & Bürgenstock & Kein Hauptthema \\
\hline 1953 & Rheinfelden & Toxikosen \\
\hline 1954 & & $\begin{array}{l}\text { Keine Jahrestagung wegen Congrès de la Fédération Internationale de } \\
\text { Gynécologie et d'Obstétrique (FIGO) in Genève }\end{array}$ \\
\hline 1955 & Baden & Fibrome des Uterus \\
\hline 1956 & Lugano & Kein Hauptthema \\
\hline 1957 & Neuchâtel & Physiologie et pathologie de la ménopause \\
\hline 1958 & Chur & $\begin{array}{l}\text { 1. Urininkontinenz bei der Frau und deren Behandlung } \\
\text { 2. Die Störung der Spermatogenese und deren Behandlung }\end{array}$ \\
\hline 1959 & Bürgenstock & $\begin{array}{l}\text { 1. Röntgenologische Beckenmessung } \\
\text { 2. Schwangerschaftsanämie }\end{array}$ \\
\hline 1960 & Fribourg & Die serologische Inkompatibilität zwischen Mutter und Kind \\
\hline 1961 & Interlaken & Les staphylococcies en obstétrique \\
\hline 1962 & Biel & Kein Hauptthema \\
\hline 1963 & La Tour-de- & \\
\hline & Peilz & Génétique humaine et intersexualité \\
\hline 1964 & $\begin{array}{l}\text { Schaffhau- } \\
\text { sen }\end{array}$ & Geburtsanalgesie und Sectionarbe \\
\hline 1965 & Porrentruy & $\begin{array}{l}\text { 1. Diabète et grossesse } \\
\text { 2. La femme et le sport }\end{array}$ \\
\hline 1966 & Lugano & $\begin{array}{l}\text { 1. Dysfunktionelle Blutungen } \\
\text { 2. Perinatale Physiologie }\end{array}$ \\
\hline 1967 & Genève & $\begin{array}{l}\text { 1. Die Therapie des Ovarialkarzinoms } \\
\text { 2. Mechanische Gefahren für das Kind unter der Geburt }\end{array}$ \\
\hline 1968 & Solothurn & $\begin{array}{l}\text { 1. Blutgerinnungsstörungen in der Gestation } \\
\text { 2. La gynécologie psychosomatique }\end{array}$ \\
\hline 1969 & Gstaad & L'accoucheur et la protection prénatale de l'enfant \\
\hline 1970 & Winterthur & Die Rolle des Gynäkologen in der Präventivmedizin \\
\hline 1971 & Crans & $\begin{array}{l}\text { 1. Behandlung des Endometrium-Karzinoms } \\
\text { 2. Immunologie vaginale } \\
\text { 3. Heteroinsemination }\end{array}$ \\
\hline 1972 & Locarno & $\begin{array}{l}\text { 1. Das Karzinom der Brust } \\
\text { 2. Adaption sexuelle de la femme et ses troubles }\end{array}$ \\
\hline 1973 & Basel & $\begin{array}{l}\text { 1. Echecs et complications des opérations en gynécologie et obstétrique } \\
\text { 2. L'exploration de la fonction ovarienne }\end{array}$ \\
\hline
\end{tabular}


1974 Luzern 1. Die Bestimmung der intrauterinen Größe und Reife des Kindes

2. Sterilität mit besonderer Berücksichtigung des Zervikalfaktors

1975 Nyon 1. Die Beeinflussung des Geburtsverlaufs

2. Gynäkologische Urologie

1976 Davos 1. Perinatale Hypoxie

2. Die Therapie der gestörten Ovarialfunktion

1977 Lugano 1. Präventivmaßnahmen in der Gynäkologie und ihre ökonomischen Aspekte

2. Opérations esthétiques et plastiques en gynécologie

1978 Biel 1. Prostaglandine und Antiprostaglandine

2. Dépistage et prophylaxie de l'accouchement prématuré idiopathique

1979 Montreux 1. Psychologie et médecine périnatale

2. Gynécologie du $3^{\mathrm{e}}$ âge

1980 St.Gallen 1. Geburtshilfliche Analgesie und Anästhesie

2. Sinn und Unsinn der Hormonbestimmungen in Geburtshilfe und Gynäkologie

1981 Genève 1. Allaitement maternel

2. Colloque interdisciplinaire sur les cancers gynécologiques (présentation de cas)

\section{Summary}

In 1905, French speaking Swiss gynaecologists founded a professional society. A few years later, their German speaking colleagues did the same. During World War I, the two societies gave a good example by forming an union. Once more the Swiss gynaecologists proved a good judgement, when they rejected, in 1938, the German demand of a confession to nationalsocialistic principles. So this compilation is also a report of the conquest of dangers.

Prof. Dr. med. Heinrich Stamm

Chefarzt der Geburtshilflich-gynäkologischen Klinik am Kantonsspital Baden

CH-5404 Baden 